\title{
Sustained drug-related reaction with eosinophilia and systemic symptoms (DRESS) triggered by low molecular weight heparins in COVID-19: management and precision diagnosis
}

\author{
Elena Minenna ${ }^{1,2,3}$, Nada Chaoul ${ }^{3}$, Maria Pia Rossi ${ }^{3}$, Lucia Giliberti ${ }^{3}$, Marcello Albanesi ${ }^{1,3}$, Eustachio Nettis ${ }^{3}$, \\ Maria Pia Foschino Barbaro², Danilo Di Bona ${ }^{3}$, Maria Filomena Caiaffa ${ }^{1}$, Luigi Macchia ${ }^{3}$
}

\begin{abstract}
${ }^{1}$ Department of Medical and Surgical Sciences, Chair of Allergology and Clinical Immunology, University of Foggia, Foggia, Italy ${ }^{2}$ Department of Medical and Surgical Sciences, Chair of Respiratory Diseases, University of Foggia, Foggia, Italy ${ }^{3}$ Department of Emergency and Organ Transplantation, School and Chair of Allergology and Clinical Immunology, University of Bari Aldo Moro, Bari, Italy

Adv Dermatol Allergol 2022; XXXIX (4): 816-818 DOI: https://doi.org/10.5114/ada.2021.109586
\end{abstract}

Low molecular weight heparins (LMWHs), particularly enoxaparin, are administered routinely to Coronavirus disease 2019 (COVID-19) patients, in order to treat or prevent the associated, possibly serious coagulation disorders [1].

Both immunoglobulin E (IgE)-mediated and non lgEmediated systemic reactions to $\mathrm{LMWH}$ s are considered rare [2-4]. Particularly, a single case of the drug-related reaction with eosinophilia and systemic symptoms (DRESS) has been documented in the literature [4].

Here we report on an elderly COVID-19 patient who developed DRESS upon prophylactic treatment with enoxaparin. Etiological diagnosis was corroborated by the lymphocyte proliferation test (LPT) [5] upon recovery. The management strategy adopted is outlined and commented.

A 95-year-old female resident of a nursing home developed acute respiratory symptoms, at the peak of the COVID-19 "first wave" in Italy in March 2020. Upon hospitalization, infection by SARS-COV2 was confirmed by nasopharyngeal swab, followed by polymerase chain reaction (PCR) detection of the Coronavirus. Chest computed tomography revealed diffuse bilateral infiltrates. At admission, blood counts were in the normal range (including eosinophils, $70 / \mu \mathrm{l}$ ). The elevated erythrocyte sedimentation rate $(87 \mathrm{~mm} / \mathrm{h})$ and C-reactive protein (18.7 mg/l) were the only abnormal laboratory values. Underlying comorbidities included type 2 diabetes and hypertension (both moderate and well controlled). Upon hospitalization, she was treated (empirically) with hy- droxychloroquine (400 mg/day) and enoxaparin (4,000 UI/day). Her conditions remained fair for 2 weeks (with no need for oxygen therapy), when she presented with a maculopapular skin eruption with a purpuric aspect and scaling, involving more than $50 \%$ of the body surface (abdomen, back, upper and lower limbs), accompanied by severe pruritus and burning sensation. Fever (> $38.5^{\circ} \mathrm{C}$ ), lymph node swelling at multiple peripheral stations and elevated serum creatinine values completed the clinical picture. The withe blood cell count reached $15,160 / \mu$ l, with eosinophil count at 1,540/ $\mu$ l. A DRESS diagnosis was established (RegiSCAR score $=7$ ) [6]. Thus, hydroxychloroquine, often associated with severe drug hypersensitivity, including DRESS [7, 8], was discontinued. Enoxaparin dosage was doubled (8,000 IU/day), in consideration of increased D-dimer values $(2.173 \mu \mathrm{g} / \mathrm{ml})$. Prednisone ( $25 \mathrm{mg} /$ day) and cetirizine $(10 \mathrm{mg} /$ day) were added to the therapy. However, a further deterioration of the clinical conditions occurred, leading to replacement of enoxaparin with the fully synthetic pentasaccharide factor Xa inhibitor fondaparinux (2.5 mg/day, subcutaneously), generally well tolerated in patients with local non-immediate cutaneous reactions to LMWHs, including enoxaparin $[9,10]$.

Management of the case according to this therapeutic schedule led to a slow resolution of maculopapular lesions, over approximately 1 month, with a substantial fever decline achieved in 2 weeks and apyrexia in 3 weeks. Finally, the eosinophil counts also declined steadily and normalized by day 30 after the emergence of the DRESS

Address for correspondence: Luigi Macchia MD, PhD, Department of Emergency and Organ Transplantation, School and Chair of Allergology and Clinical Immunology, University of Bari - Aldo Moro, Piazza Giulio Cesare, 70124 Bari, Italy, e-mail: luigi.macchia@uniba.it Received: 29.05.2021, accepted: 25.07.2021. 
eruption. Prednisone and fondaparinux were then discontinued.

A few days later, the patient was discharged, upon double successive negative nasopharyngeal swab.

Precision diagnosis: Following a 7-day corticosteroid wash-out period, a blood sample was obtained from the patient (fully recovered; at home). Peripheral blood mononuclear cells were isolated by gradient centrifugation $\left(800 \times\right.$ g, 45’) on Lympholyte ${ }^{\oplus}$ (Cedarlane, EuroClone, Milan, Italy), upon plasma removal and suspension of the blood cellular moiety in Dulbecco's phosphate buffered saline. Mononuclear cells were maintained in Dulbecco's modified Eagle's medium, with 10\% autologous serum $(\mathrm{v} / \mathrm{v})$, with streptomycin $(100 \mu \mathrm{g} / \mathrm{ml})$, at $37^{\circ} \mathrm{C}$, in a $5 \% \mathrm{CO}_{2}$, vapour-saturated atmosphere, in $64 \mathrm{~cm}^{2}$ glass Petri dishes, for 4 days, in order to allow clearance of the monocyte-macrophage component. Successively, micro-cultures were generated with the resulting purified lymphocytes $\left(6 \times 10^{4}\right.$ cells in $\left.200 \mu \mathrm{l}\right)$ and employed for carrying out LPT for enoxaparin and fondaparinux, respectively, under culturing conditions as above. LPT was performed essentially as described [11]. Briefly, triplicate micro-cultures were incubated with the two drugs, respectively, at three different ten-fold concentrations: therapeutic concentration (TC; calculated on the drug distribution volume), TC/10 and TCX10 (defect and excess concentration, respectively). The distribution volume and the TC were $5.24 \mathrm{I}$ and $15 \mathrm{mg} / \mathrm{l}$, for enoxaparin [12], and $9 \mathrm{I}$ and $0.27 \mathrm{mg} / \mathrm{l}$ for fondaparinux [13]. Triplicate micro-cultures incubated with phytohemagglutinin-M (from Phaseolus vulgaris; $2.25 \mathrm{\mu g} / \mathrm{ml}$ ) and the medium alone served as the positive and the negative control, respectively. Following 4-day incubation with the drugs, lymphocyte proliferation was assessed upon inclusion of the non-radioactive thymidine analogue 5-bromo-2'-deoxyuridine (BrdU; $100 \mu \mathrm{M})$, in the micro-cultures, for $2 \mathrm{~h}$.

A

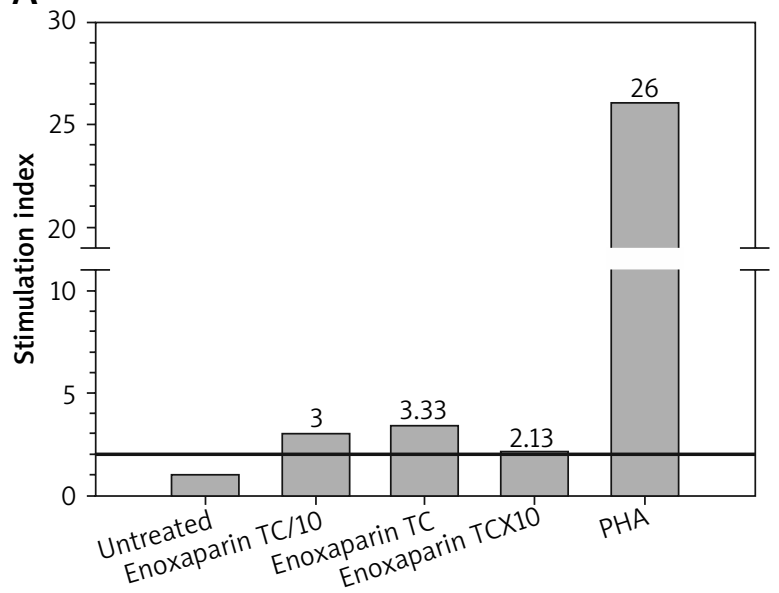

Incorporation of the nucleotide in proliferating cells was evaluated by an anti-BrdU monoclonal antibody (7.5 U/ $\mathrm{ml}$; from Roche Diagnostics $\mathrm{GmbH}$, Mannheim, Germany) [14]. The LPT is deemed positive when the proliferation rate of any of the three concentrations tested compared to the negative control (stimulation index) equals or exceeds $2[5,11]$.

The assay revealed that not only enoxaparin (as suspected), but also fondaparinux induced significant lymphocyte proliferation (Figure 1).

LMWHs, including enoxaparin, have been involved mainly in local delayed hypersensitivity reactions. These reactions are not very infrequent and often mild or moderate. Fondaparinux appears to be tolerated by patients with previous delayed local reactions to LMWHs [8, 10].

Systemic severe delayed reactions, particularly DRESS, are rarer. To our knowledge, this is the second report of a DRESS associated to exposure to enoxaparin and the first one of DRESS associated to fondaparinux. Moreover, by LPT, we showed that the two LMWHs cross-reacted with each other, making fondaparinux an unlikely alternative to enoxaparin in the case of severe delayed systemic reactions (in spite of the fully synthetic structure).

Thus, a full-blown DRESS occurred in an elderly COVID-19 patient (with important comorbidities). This led to discontinuation of the probably useless hydroxychloroquine administration, in fear of a possible causative role. Enoxaparin was then suspected and discontinued. A sustained high dosage corticosteroid treatment was undertaken and the patient eventually recovered from both COVID-19 and DRESS, and survived. Probably correct was the choice of maintaining anti-coagulation by LMWHs. Enoxaparin was indeed replaced with fondaparinux, in the belief that the latter drug had a less allergenic profile. Although, in retrospect, LPT proved it wrong, anticoagulation was probably instrumental in obtaining the healing.

B

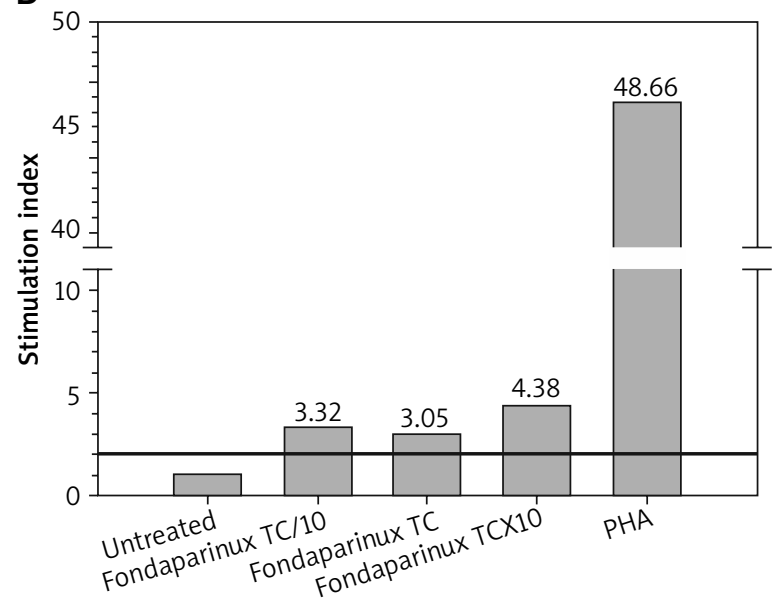

Figure 1. LPT results for enoxaparin (A) and fondaparinux (B). Lymphocyte micro-cultures were exposed to the culprit drugs at therapeutic concentration (TC), TC/10 and TCX10, respectively. The test was consistently positive (stimulation index $\geq 2$ ), for all three concentrations for both drugs. Lymphocyte response to the mitogen phytohemagglutinin-M (PHA) was openly valid 


\section{Conflict of interest}

The authors declare no conflict of interest.

\section{References}

1. Tang N, Bai H, Chen X, et al. Anticoagulant treatment is associated with decreased mortality in severe coronavirus disease 2019 patients with coagulopathy. J Thromb Haemost 2020; 18: 1094-9.

2. Harr T, Scherer K, Tsakiris DA, Bircher AJ. Immediate type hypersensitivity to low molecular weight heparins and tolerance of unfractionated heparin and fondaparinux. Allergy 2006; 61: 787-8.

3. Cesana P, Scherer K, Bircher AJ. Immediate type hypersensitivity to heparins: two case reports and a review of the literature. Int Arch Allergy Immunol 2016; 171: 285-9.

4. Ronceray S, Dinulescu M, Le Gall F, et al. Enoxaparin-induced DRESS syndrome. Case Rep Dermatol 2012; 4: 233-7.

5. Porebski G, Gschwend-Zawodniak A, Pichler WJ. In vitro diagnosis of T-cell mediated drug allergy. Clin Exp Allergy 2011; 41: 461-70

6. Kardaun SH, Sekula P, Valeyrie-Allanore L, et al. Drug reaction with eosinophilia and systemic symptoms (DRESS): an original multisystem adverse drug reaction. Results from the prospective RegiSCAR study. Br I Dermatol 2013; 169: 1071-80.

7. Volpe A, Marchetta A, Caramaschi P, et al. Hydroxychloroquine-induced DRESS syndrome. Clin Rheumatol 2008; 27: 537-9.

8. Gelincik A, Brockow K, Çelik GE, et al. Diagnosis and management of the drug hypersensitivity reactions in Coronavirus disease 19: an EAACI Position Paper. Allergy 2020; 75: 2775-93.

9. Schindewolf $\mathrm{M}$, Scheuermann J, Kroll H, et al. Low allergenic potential with fondaparinux: results of a prospective investigation. Mayo Clin Proc 2010; 85: 913-9.

10. Tan E, Thompson G, Ekstrom C, Lucas M. Non-immediate heparine and heparinoid cutaneous allergic reactions: a role for fondaparinux. Intern Med J 2018; 48: 73-7.

11. Albanesi M, Di Girolamo A, Aresta V, et al. Delayed allergy to acyclovir revealed by lymphocyte proliferation test. Adv Dermatol Allergol 2018; 35: 527-9.

12. Bruno R, Baille P, Retout S, et al. Population pharmacokinetics and pharmacodynamics of enoxaparine in unstable angina and non-ST-segment elevation myocardial infarction. Br J Clin Pharmacol 2003; 56: 407-14.

13. Donat F, Duret JP, Santoni A, et al. The pharmacokinetics of fondaparinux sodium in healthy volunteers. Clin Pharmacokinet 2002; 41 Suppl. 2: 1-9.

14. Heil J, Reifferscheid G. Detection of mammalian carcinogens with an immunological DNA synthesis-inhibition test. Carcinogenesis 1992; 13: 2389-94. 